\title{
Low compositions of human toll-like receptor 7/8-stimulating RNA motifs in the MERS-CoV, SARS-CoV and SARS-CoV-2 genomes imply a substantial ability to evade human innate immunity
}

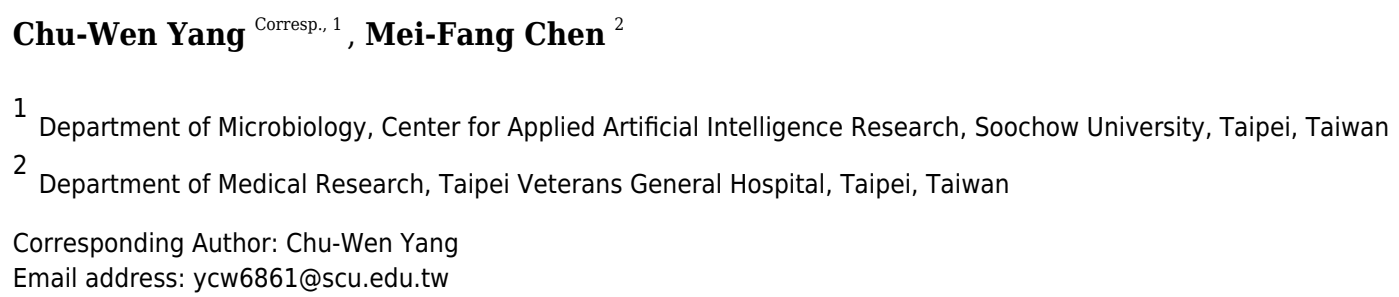

Background. The innate immune system especially Toll-like receptor (TLR) $7 / 8$ and the interferon pathway, constitutes an important first line of defense against single-stranded RNA viruses. However, large-scale, systematic comparisons of the TLR 7/8-stimulating potential of genomic RNAs of single-stranded RNA viruses are rare. In this study, a computational method to evaluate the human TLR 7/8-stimulating ability of singlestranded RNA virus genomes based on their human TLR 7/8-stimulating trimer compositions was used to analyze 1,002 human coronavirus genomes. Results. The human TLR 7/8-stimulating potential of coronavirus genomic (positive strand) RNAs followed the order of NL63-CoV > HKU1-CoV >229E-CoV $\cong$ OC63-CoV > SARS-CoV-2 > MERS-CoV > SARS-CoV. These results suggest that among these coronaviruses, MERS-CoV, SARS-CoV and SARS-CoV-2 may have a higher ability to evade the human TLR 7/8mediated innate immune response. Analysis with a logistic regression equation derived from human coronavirus data revealed that most of the 1,762 coronavirus genomic (positive strand) RNAs isolated from bats, camels, cats, civets, dogs and birds exhibited weak human TLR 7/8-stimulating potential equivalent to that of the MERS-CoV, SARS-CoV and SARS-CoV-2 genomic RNAs. Conclusions. Prediction of the human TLR 7/8stimulating potential of viral genomic RNAs may be useful for surveillance of emerging coronaviruses from nonhuman mammalian hosts. 
2 Low compositions of human toll-like receptor 7/8-

3 stimulating RNA motifs in the MERS-CoV, SARS-CoV

4 and SARS-CoV-2 genomes imply a substantial ability

5 to evade human innate immunity

6

7

8 Chu-Wen Yang ${ }^{1 *}$, Mei-Fang Chen ${ }^{2}$

9

10

${ }^{1}$ Department of Microbiology, Center for Applied Artificial Intelligence Research, Soochow

University, Taipei 111, Taiwan

${ }^{2}$ Department of Medical Research, Taipei Veterans General Hospital, Taipei 112, Taiwan

Corresponding Author:

17 Chu-Wen Yang *

No. 70, Linxi Rd., Shilin Dist., Taipei City 111002, Taiwan 


\section{Abstract}

23

Background. The innate immune system especially Toll-like receptor (TLR) 7/8 and the interferon pathway, constitutes an important first line of defense against single-stranded RNA viruses. However, large-scale, systematic comparisons of the TLR 7/8-stimulating potential of genomic RNAs of single-stranded RNA viruses are rare. In this study, a computational method to evaluate the human TLR 7/8-stimulating ability of single-stranded RNA virus genomes based on their human TLR 7/8-stimulating trimer compositions was used to analyze 1,002 human coronavirus genomes.

Results. The human TLR 7/8-stimulating potential of coronavirus genomic (positive strand) RNAs followed the order of NL63-CoV $>$ HKU1-CoV $>229 \mathrm{E}-\mathrm{CoV} \cong \mathrm{OC} 63-\mathrm{CoV}>$ SARS-CoV$2>$ MERS-CoV $>$ SARS-CoV. These results suggest that among these coronaviruses, MERSCoV, SARS-CoV and SARS-CoV-2 may have a higher ability to evade the human TLR 7/8mediated innate immune response. Analysis with a logistic regression equation derived from human coronavirus data revealed that most of the 1,762 coronavirus genomic (positive strand) RNAs isolated from bats, camels, cats, civets, dogs and birds exhibited weak human TLR 7/8stimulating potential equivalent to that of the MERS-CoV, SARS-CoV and SARS-CoV-2 genomic RNAs.

Conclusions. Prediction of the human TLR 7/8-stimulating potential of viral genomic RNAs may be useful for surveillance of emerging coronaviruses from nonhuman mammalian hosts.

Keywords: SARS-CoV-2; Toll-like receptor 7/8; immunostimulating RNA motifs 
46 Introduction

47

48

49

50

51

52

53

54

The novel coronavirus disease 2019 (COVID-19) caused by severe acute respiratory syndrome coronavirus 2 (SARS-CoV-2) has developed into a global pandemic (Li et al., 2020; Zheng, 2020). Understanding the virology of SARS-CoV-2 and the development of therapeutics to treat viral infection is an urgent need (Jin et al., 2020; Zhou et al., 2020).

The diversity of rapidly evolved single-stranded RNA virus genome sequences among virus strains may affect viral infectivity or pathogenicity in many ways. For example, diversity in viral genome sequences may lead to changes in viral protein sequences and, consequently, changes in viral protein activity that may affect viral replication, transmission (interactions with the receptors for the virus) and antigenicity (interactions with components of the host adaptive immune system). These issues have been extensively addressed by various studies of viral protein functions (Elfiky, 2020; Ou et al., 2020; Xia et al., 2020). The second effect of RNA virus genome diversity is differences in protein short linear motif (SLiM) compositions among virus strains. Several viruses have been reported to interact with host cell components through viral protein SLiMs (that mimic host protein SLiMs) to facilitate manipulation of host cellular networks (Yang, 2012a; Via et al., 2015). The third effect of RNA virus genome diversity is differences in genetic codon compositions. Yang et al. (2020) demonstrated that the proportions of human-specific slow codons and slow di-codons in SARS-CoV and SARS-CoV-2 are lower than those in other coronaviruses. The low proportions of slow codons and slow di-codons in SARS-CoV and SARS-CoV-2 coding sequences may lead to a protein synthesis rate faster than

67 that of other coronaviruses. The fourth effect of RNA virus genome diversity is differences in the ability to interact with components of the host innate immune system. Mammalian Toll-like 
69 receptors (TLRs) 7 and 8 are usually present in endosomal compartments, where they detect viral 70 or endogenous single-stranded RNAs (Streicher and Jouvenet, 2019; Vierbuchen et al., 2019).

71 In the past, many studies have focused on developing TLR7 agonists to activate host

72 inflammatory responses to cope with RNA virus infections (Alharbi et al., 2020; Shah et al.,

73 2016). For example, TLR7 agonists were shown to provide protection against influenza A virus-

74 induced morbidity in mice (To et al., 2019). TLR7 was also proposed to be a potential

75 therapeutic target for COVID-19 (Bonam et al., 2020; Poulas et al., 2020). For example, the use

76 of imiquimod was suggested for the management of COVID-19 (Angelopoulou et al., 2020). On

77 the other hand, studies have found that people with TLR7 gene variants exhibit different disease

78 severities after SARS-CoV-2 infection (Anastassopoulou et al., 2020; Lee et al., 2020;

79 Mukherjee et al., 2020). For example, epidemiological investigations found that SARS-CoV-2

80 affects women less severely than men (Conti et al., 2020; van der Made et al., 2020). However,

81 little is known about the potential of coronaviruses to evade detection by TLR 7/8.

82 Several studies have indicated that ribonucleotide compositions of RNAs are crucial for

83 TLR 7/8 stimulation (Forsbach et al., 2011; Green et al., 2012). Heil et al. (2004) found that G-

84 and U-rich single stranded RNA oligonucleotides derived from human immunodeficiency virus-

851 (HIV-1) stimulate dendritic cells (DCs) and macrophages to secrete interferon and

86 proinflammatory cytokines. Subsequently, several studies found that TLR7 recognizes G- and U-

87 rich motifs in single stranded RNAs (Diebold et al. 2006; Forsbach et al. 2007). Moreover,

88 certain GU- or AU-rich RNA sequences were described to induce human TLR7- and TLR8-

89 mediated immune responses (Forsbach et al. 2011; Krüger et al. 2015; Zhang et al. 2018).

90 Kosuge et al. (2020) found that there is a bias to the mutations occurring in SARS-CoV-2

91 variants, with a preference for cytosine (C) to uracil (U) mutations. The degree of the increase in 
92 U nucleotides in SARS-CoV-2 variants correlates with enhanced production of cytokines, such

93 as TNF- $\alpha$ and IL-6, in cell lines. Overall, these results indicate that genome sequence variations

94 in RNA viruses (such as coronaviruses) may induce different degrees of human TLR7- and

95 TLR8-mediated immune responses and, as a consequence, result in different degrees of disease

96 severity. Therefore, genome sequence diversity may endow single-stranded RNA viruses with

97 different abilities to evade the host TLR 7/8-mediated innate immune responses.

$98 \quad$ Yang et al. (2012b) developed a computational method to evaluate the human TLR 7/8-

99 stimulating ability of single-stranded RNA virus genomes based on their human TLR 7/8-

100 stimulating triribonucleotide compositions. In this study, the method was applied to analyze the

101 RNA genomes of coronaviruses infecting humans. A logistic regression model was proposed for

102 prediction of coronaviruses (from nonhuman animals) with low human TLR 7/8-stimulating

103 activity (and, as a consequence, $\mathrm{n}$ higher potential to evade the human TLR 7/8-mediated innate

104 immune responses).

105

106

107

108

\section{Materials \& Methods}

\section{Data collection}

The complete (full-length) genome sequences of 1,002 coronaviruses infecting humans

110 (including 22 human coronavirus 229E (HCoV-229E), 61 human coronavirus NL63 (HCoV-

111 NL63), 26 human coronavirus HKU1 (HCoV-HKU1), 139 human coronavirus OC43 (HCoV-

112 OC43), 63 severe acute respiratory syndrome coronavirus (SARS-CoV), 121 Middle East

113 respiratory syndrome coronavirus (MERS-CoV), 432 severe acute respiratory syndrome

114 coronavirus 2 (SARS-CoV-2)) and 138 unclassified coronaviruses (Other) were retrieved from

115 the Virus Pathogen Resource (ViPR, https:/www.viprbrc.org/) (Pickett et al., 2012) and

116 analyzed in this study (Table S1). In addition, 1,762 complete (full-length) genomic sequences of 
117 coronaviruses from six nonhuman mammalians (bat, camel, cat, civet, dog and pig) and avian

118 hosts were retrieved from the Virus Pathogen Resource and used for analysis (Table S2).

119 Ninety-five oligoribonucleotides (ORNs) and 39 ribonucleotide tetramers with

120 experimentally validated human TLR 7/8-stimulating activity were identified from 17 research

121 reports (Table S3). The sequences of TLR 7 proteins from different organisms exhibit high

122 variations. For example, the sequence identity of TLR 7 proteins from humans and mice is $81 \%$.

123 The preferences for ligand nucleotide compositions of TLRs from different organisms might be

124 different. Since the experiments validating the TLR 7/8-stimulating activity of these ORN

125 sequences were conducted using human cells, the TLR-stimulating triribonucleotide composition

126 and TLR-stimulating scores described in this study should be considered to be specific for

127 human TLR 7/8.

128

\section{Weighted triribonucleotide compositions of single-stranded RNA virus genomes}

130

A method to evaluate the human TLR 7/8-stimulating ability of single-stranded RNA virus genomes based on their human TLR 7/8-stimulating triribonucleotide compositions was developed by Yang et al. (2012b). The $4^{3}=64$ possible trimers are labeled as $X_{1}, X_{2}, \ldots, X_{64}$.

Each trimer frequency $f_{X i}$ is defined as

$f_{X i}=c_{X i} / l, i=1,2, \ldots, 64, \quad(1)$,

135 where $c_{X i}$ is the number of trimer $X i$ and $l$ is the total number of trimers. The trimer weights $w_{X i}$

136 were computed using the following formula:

$137 w_{X i}=\log _{10}\left(\frac{f_{X i}}{1 / 64}\right)=\left\{\begin{array}{l}w_{X i}^{+} \text {if } f_{X i}>\frac{1}{64} \\ w_{X i}^{-} \text {if } f_{X i}<\frac{1}{64}\end{array}\right.$

138 where $w_{X i}^{+}$and $w_{X i}^{-}$are the weights of overrepresented and underrepresented human TLR 7/8- 
140 stimulating ORN sequences is greater than 1/64 (the expected value of a random distribution),

141 the trimer is considered to be human TLR 7/8 stimulatory. Otherwise, the trimer is considered to

142 be nonhuman TLR 7/8-stimulatory. Each trimer is assigned a weight based on the logarithm of

143 its relative frequency in the human TLR 7/8-stimulating ORN sequences (Fig. 1).

144 For any individual RNA virus genome, the positive and negative weighted trimer

145 compositions were calculated and are referred to as Score S and Score N, respectively, these

146 scores are collectively referred to as the human TLR 7/8-stimulating scores. Score S for

147 stimulating trimers was calculated as

$148 \quad$ Score $\mathrm{S}=\Sigma\left(c_{X i} \cdot w_{X i}^{+}\right) / l \quad(3)$,

149 and Score $\mathrm{N}$ for nonstimulating trimers was calculated as

150 Score $\mathrm{N}=\Sigma\left(c_{X i} \cdot w_{X i}^{-}\right) / l \quad(4)$,

151 where $c_{X i}$ is the number of trimer $X i$ that appear in the viral genomic RNA (with $i=1, \ldots 64$ ). $l$ is

152 the total number of trimers in the viral genomic RNA. Higher Score S and lower Score N values

153 indicates greater numbers of human TLR 7/8-stimulating triribonucleotides in the viral RNA

154 genome and implies that stronger human TLR 7/8-mediated innate immunity may be induced by

155 this viral RNA. Conversely, lower Score S and higher Score N values indicate greater numbers

156 of human TLR 7/8 nonstimulating triribonucleotides in the viral RNA genome and, as a

157 consequence, a higher potential for evasion of the human TLR 7/8-mediated innate immune

158 responses (Fig. 1).

159

160 Data analysis

161 Data manipulation was performed with Perl scripts written by the author. The heatmap,

162 stripchart of Logit P values and scatter plots of Score S and Score N values were plotted using 
163 the ggplot2 package of $\mathrm{R}$ (the R package for statistical computing). Logistic regression was

164 conducted with the glm function of R. Linear discriminant analysis (LDA) and quadratic

165 discriminant analysis (QDA) were performed with the lda and qda functions, respectively, in the

166 MASS package of R. Naive Bayes and support vector machine (svmLinear, svmPoly and 167 svmRadial) classifiers in the caret package of $\mathrm{R}$ were used.

168

169

\section{Tenfold cross-validation}

The cross-validation test is well-known and has been used in many computation-based

171

172

173

174

175

176

177

178

179

180

181

182

183

184

185

studies (Le et al., 2017; Le et al., 2019a; Le et al., 2019b). The weighted triribonucleotide

compositions of coronavirus genomes were randomly split into 10 subsets. Ten pairs of training

(90\% data for model building) and test (10\% data for model evaluation) data were combined

from the 10 subsets and used to perform 10 -fold cross validations. For each test run, a training

set was used to train a model, and the model was then tested using the test set. All methods

(logistic regression, LDA, QDA, naive Bayes, svmLinear, svmPoly and svmRadial) were used to

perform 10-fold cross validations. Sensitivity was computed with the following formula:

sensitivity $=$ true positive $/$ (true positive + false negative). Specificity was computed with the

following formula: specificity $=$ true negative $/($ true negative + false positive $)$. Accuracy was computed with the following formula: accuracy $=($ true positive + true negative $) /$ total number of samples.

\section{Analysis of coronaviruses from nonhuman animals}

Seven models derived from the 7 methods (logistic regression, LDA, QDA, naive Bayes, svmLinear, svmPoly and svmRadial) were used to analyze data of coronaviruses from nonhuman 
186 187 188

193

194

195

196

197 198

199

200

201

202

203

204

205

206

207

208

animals. All 7 methods were selected for binary classification to distinguish human

coronaviruses causing common colds and severe acute respiratory syndromes. Using the results of logistic regression as a standard, the overall agreements between the results of the logistic regression model and those of the other 6 models were computed. The overall agreements were computed by the following formula: (true positive + true negative) / total number of samples.

\section{Results}

\section{Compositions of triribonucleotides in genomes of coronaviruses infecting humans}

The similarity of triribonucleotide compositions was not consistent with the similarity of genome sequences (phylogenetic analysis in Fig. 2A). The overall triribonucleotide compositions of genomic (plus) strand and complementary (minus) strand RNAs of coronaviruses infecting humans are shown in Fig. 2B. These results indicate that triribonucleotide compositions provide novel information that cannot be revealed by phylogenetic analysis.

\section{Human TLR 7/8-stimulating potential of human coronavirus genomes}

The human TLR 7/8-stimulating scores of coronavirus genomic (positive strand) RNAs are shown in Fig. 3. The human TLR 7/8-stimulating potential of coronavirus genomic (positive strand) RNAs followed the order of NL63-CoV $>\mathrm{HKU1-CoV}>229 \mathrm{E}-\mathrm{CoV} \cong \mathrm{OC} 63-\mathrm{CoV}>$ SARS-CoV-2 > MERS-CoV > SARS-CoV. The human TLR 7/8-stimulating scores of the complementary (negative) strand of coronavirus genomic RNAs (replication intermediates) are shown in Fig. 4. The human TLR 7/8-stimulating potential of the complementary (negative) 
210 strand of coronavirus genomic RNAs followed the order of SARS-CoV-2 > SARS-CoV >229E-

$211 \mathrm{CoV}>\mathrm{MERS}-\mathrm{CoV}>\mathrm{NL63}-\mathrm{CoV} \cong \mathrm{OC} 63-\mathrm{CoV}>\mathrm{HKU} 1-\mathrm{CoV}$. The highest Score $\mathrm{S}$ value of the 212 complementary (negative) strand of SARS-CoV-2 genomic RNA wass 0.0813 which was less 213 than the minimum Score S value of SARS-CoV genomic (positive strand) RNA. Greater 214 numbers of human TLR 7/8-stimulating triribonucleotides were found in the genomic (positive 215 strand) RNAs than in the complementary strand RNAs of all coronaviruses analyzed. These 216 results suggest a greater potential for human TLR 7/8-stimulating activity in the early stage of 217 viral infection than the complementary (negative) strand RNAs produced during coronavirus 218 replication stage.

219

220

\section{A logistic regression model for predicting the human TLR 7/8-stimulating potential}

To predict the human TLR 7/8-stimulating potential of coronaviruses, a logistic regression 222 model was constructed as follows:

223

$\operatorname{logit}(p)=\log (p / 1-p)=\beta_{0}+\beta_{1} \cdot \mathrm{S}+\beta_{2} \cdot \mathrm{N}+\beta_{3} \cdot \mathrm{Sr}+\beta_{4} \cdot \mathrm{Nr}$

$\mathrm{S}$ and $\mathrm{N}$ are the Score $\mathrm{S}$ and Score $\mathrm{N}$ values for the positive strand of a viral genome sequence.

$\mathrm{Sr}$ and $\mathrm{Nr}$ are the Score $\mathrm{S}$ and Score $\mathrm{N}$ values for the negative strand of a viral genome sequence. 229E-CoV, OC43-CoV, NL63-CoV and HKU1-CoV were used as the highly stimulating group. MERS-CoV, SARS-CoV and SARS-CoV-2 were used as the poorly stimulating group. After the model selection procedure, the following model was selected as the final model:

$\operatorname{logit}(p)=\log (p / 1-p)=\beta_{0}+\beta_{1} \cdot \mathrm{S}$

230 The results of 10-fold cross-validation are shown in Table 1. The averages of the intercepts and 231 coefficients of the 10 models were used to construct the logistic regression model as follows:

$232 \operatorname{logit}(p)=\log (p / 1-p)=-71.01+730.49 \cdot \mathrm{S}(7)$ 
233 The logistic regression model using all data is:

234

$\operatorname{logit}(p)=\log (p / 1-p)=-69.05+710.36 \cdot \mathrm{S}$

235

236

\section{Comparison with other methods}

237

Six methods were used to validate the results of logistic regression. The results of 10 -fold

238

239

240

241

242

243

244

245

246

247

248

249

250

251

252

253

254

255

256

cross-validations using linear discriminant analysis (LDA), quadratic discriminant analysis

(QDA), naive Bayes and three support vector machine classifiers (linear, polynomial and radial)

were the same as those using the logistic regression model (Table 1$)$. The high values $(\cong 1)$ of sensitivity, specificity and accuracy may be due to the almost complete separation of the Score $\mathrm{S}$ values of the two groups. These results are consistent with the data shown in Fig. 3.

\section{Discussion}

The innate immune system especially the TLR 7/8-interferon pathway constitutes an important first line of defense against single-stranded RNA viruses (Vierbuchen et al. 2019;

Kikkert 2020; Nelemans and Kikkert 2019). Conversely, RNA viruses have evolved multiple strategies to evade host innate immune responses to increase the success rate of infection.

Several molecular mechanisms by which positive-sense single-stranded RNA viruses evade innate immune responses have been identified (Ye et al. 2020). Since interferons have great protective effects during early viral infection, evasion of the immune response may have differential effects on the clinical outcome of viral disease (de Marcken et al. 2019). Therefore, prediction of the human TLR 7/8-stimulating activities of genomic RNAs of single-stranded RNA viruses provides a method to evaluate the risk potential posed by emerging single-stranded RNA viruses. 
The results of this study suggest that the genomic (positive strand) RNAs of MERS-CoV,

258 SARS-CoV and SARS-CoV-2 are composed of low proportions of human TLR 7/8-stimulating

259 triribonucleotides. The weak human TLR 7/8-stimulating potential of the genomic (positive

260 strand) RNAs of MERS-CoV, SARS-CoV and SARS-CoV-2 may lead to a high ability to evade

261 the human TLR 7/8-mediated innate immune responses during the initial stage of viral infection.

262 In contrast, the strong human TLR 7/8-stimulating potential of the genomic (positive strand)

263 RNAs of 229E-CoV, NL63-CoV, OC43-CoV and HKU1-CoV may confer a high probability of

264 triggering strong human TLR 7/8-mediated innate immune responses. Different strengths of TLR

265 7/8-mediated innate immune responses during the initial stage of viral infection may lead to

266 different clinical outcomes of the disease (Frieman and Baric 2008; Wong et al. 2016; Yokota et

267 al. 2010). Evaluation of the human TLR 7/8-stimulating potential of viral genomic RNAs may be

268 useful for surveillance of emerging coronaviruses.

269 Coronavirus infections have been considered novel emerging zoonotic diseases (Streicher

270 and Jouvenet 2019; Salata et al. 2019; Menachery et al. 2019). Evaluating the risk potential

271 posed by zoonotic coronaviruses is necessary. The logistic regression model constructed in this

272 study can be used to evaluate the human TLR 7/8-stimulating potential of genomic RNAs of

273 coronaviruses from other mammals and birds. For example, the human TLR 7/8-stimulating

274 potential of 1,361 coronavirus genomic (positive strand) RNAs from six mammalian (bat, camel,

275 cat, civet, dog and pig) and avian hosts were computed using the logistic regression model

276 (equation 7). Logit $\mathrm{P} \cong 1$ indicates a human TLR 7/8-stimulating ability equivalent to that of

277 general human coronavirus (229E-CoV, NL63-CoV, OC43-CoV and HKU1-CoV) genomic

278 RNAs. Logit $\mathrm{P} \cong 0$ indicates weak human TLR 7/8-stimulating potential equivalent to that of the

279 highly pathogenic coronavirus (MERS-CoV, SARS-CoV and SARS-CoV-2) genomic RNAs. As 
280 shown in Fig. 5, many of the coronavirus genomic (positive strand) RNAs from bats, camels, 281 cats, civets and pigs exhibit weak human TLR 7/8-stimulating potential equivalent to that of 282 highly pathogenic coronavirus (MERS-CoV, SARS-CoV and SARS-CoV-2) genomic RNAs. 283 Six methods were used for comparison with the logistic regression model. Using the results of 284 logistic regression as a standard, the overall agreements between results of logistic regression 285 model and those of the other 6 models are shown in Table 2. Most of the analysis results (except 286 the results from the SVM radial classifier) were consistent with the prediction of the logistic 287 regression model. The predictions obtained using the logistic regression model proposed in this 288 study suggest that the routes and risks of contact with those animals (the natural reservoirs of 289 animal coronaviruses) should be addressed. This observation may be an important key point to 290 prevent the outbreak of emerging infectious diseases.

291

292

293

294 295 296 297 298 299

300 301

302

303

304

\section{Conclusions}

The results of this study suggest that MERS-CoV, SARS-CoV and SARS-CoV-2 may have a relatively low human TLR 7/8-stimulating potential and relatively high ability to evade human TLR 7/8-mediated innate immune responses. Prediction of the human TLR 7/8-stimulating potential of viral genomic RNAs may be useful for surveillance of emerging coronaviruses from nonhuman animal hosts.

\section{References}

Alharbi AS, Garcin AJ, Lennox KA, Pradeloux S, Wong C, Straub S, Valentin R, Pépin G, Li HM, Nold MF, Nold-Petry CA, Behlke MA, Gantier MP (2020). Rational design of antisense oligonucleotides modulating the activity of TLR7/8 agonists. Nucleic Acids Res 48:7052-7065. 
305 Anastassopoulou C, Gkizarioti Z, Patrinos GP, Tsakris A (2020). Human genetic factors

306 associated with susceptibility to SARS-CoV-2 infection and COVID-19 disease severity. Hum

307 Genomics 14:40.

308 Angelopoulou A, Alexandris N, Konstantinou E, Mesiakaris K, Zanidis C, Farsalinos K, Poulas

309 K (2020). Imiquimod - A toll like receptor 7 agonist - Is an ideal option for management of

310 COVID 19. Environ Res 188:109858.

311 Bonam SR, Kaveri SV, Sakuntabhai A, Gilardin L, Bayry J (2020). Adjunct Immunotherapies

312 for the Management of Severely Ill COVID-19 Patients. Cell Rep Med 1:100016.

313 Conti P, Younes A (2020). Coronavirus COV-19/SARS-CoV-2 affects women less than men:

314 clinical response to viral infection. J Biol Regul Homeost Agents 34:339-343.

315 de Marcken M, Dhaliwal K, Danielsen AC, Gautron AS, Dominguez-Villar M (2019). TLR7 and

316 TLR8 activate distinct pathways in monocytes during RNA virus infection. Sci Signal 12:

317 eaaw1347.

318 Diebold SS, Massacrier C, Akira S, Paturel C, Morel Y, Reis e Sousa C (2006). Nucleic acid

319 agonists for Toll-like receptor 7 are defined by the presence of uridine ribonucleotides. Eur $J$

$320 \quad$ Immunol 36:3256-3267.

321 Elfiky AA (2020). Ribavirin, Remdesivir, Sofosbuvir, Galidesivir, and Tenofovir against SARS-

322 CoV-2 RNA dependent RNA polymerase (RdRp): A molecular docking study. Life Sci 253:

$323 \quad 117592$.

324 Forsbach A, Nemorin JG, Montino C, Müller C, Samulowitz U, Vicari AP, Jurk M, Mutwiri GK,

325 Krieg AM, Lipford GB, Vollmer J (2008). Identification of RNA sequence motifs stimulating 326 sequence-specific TLR8-dependent immune responses. J Immunol 180:3729-3738. 
327 Forsbach A, Samulowitz U, Völp K et al. (2011). Dual or triple activation of TLR7, TLR8, 328 and/or TLR9 by single-stranded oligoribonucleotides. Nucleic Acid Ther 21: 423-436.

329 Frieman M, Baric R (2008). Mechanisms of severe acute respiratory syndrome pathogenesis and 330 innate immunomodulation. Microbiol Mol Biol Rev 72: 672-685.

331 Green NM, Moody KS, Debatis M, Marshak-Rothstein A (2012). Activation of autoreactive B 332 cells by endogenous TLR7 and TLR3 RNA ligands. J Biol Chem 287: 39789-39799.

333 Heil F, Hemmi H, Hochrein H, Ampenberger F, Kirschning C, Akira S, Lipford G, Wagner H, 334 Bauer S (2004). Species-specific recognition of single-stranded RNA via toll-like receptor 7 335 and 8. Science 303:1526-1529.

336 Jin Y, Yang H, Ji W et al. (2020). Virology, Epidemiology, Pathogenesis, and Control of 337 COVID-19. Viruses 12: 372.

338 Kikkert M (2020). Innate Immune Evasion by Human Respiratory RNA Viruses. J Innate Immun 339 12: 4-20.

340 Kosuge M, Furusawa-Nishii E, Ito K, Saito Y, Ogasawara K (2020). Point mutation bias in 341 SARS-CoV-2 variants results in increased ability to stimulate inflammatory responses. Sci Rep $342 \quad \mathbf{1 0}: 17766$.

343 Krüger A, Oldenburg M, Chebrolu C, Beisser D, Kolter J, Sigmund AM, Steinmann J, Schäfer S,

344 Hochrein H, Rahmann S, Wagner H, Henneke P, Hornung V, Buer J, Kirschning CJ (2015).

345 Human TLR8 senses UR/URR motifs in bacterial and mitochondrial RNA. EMBO Rep $346 \quad 16: 1656-1663$.

347 Le NQ, Ho QT, Ou YY (2017). Incorporating deep learning with convolutional neural networks 348 and position specific scoring matrices for identifying electron transport proteins. J Comput 349 Chem 38:2000-2006. 
350 Le NQK, Yapp EKY, Yeh HY (2019a). ET-GRU: using multi-layer gated recurrent units to 351 identify electron transport proteins. BMC Bioinformatics 20:377.

352 Le NQK, Yapp EKY, Nagasundaram N, Chua MCH, Yeh HY (2019b). Computational

353 identification of vesicular transport proteins from sequences using deep gated recurrent units 354 architecture. Comput Struct Biotechnol J 17:1245-1254.

355 Lee IH, Lee JW, Kong SW (2020). A survey of genetic variants in SARS-CoV-2 interacting 356 domains of ACE2, TMPRSS2 and TLR3/7/8 across populations. Infect Genet Evol 85:104507.

357 Li H, Zhou Y, Zhang M, Wang H, Zhao Q, Liu J (2020). Updated Approaches against SARS358 CoV-2. Antimicrob Agents Chemother 64: e00483-20.

359 Menachery VD, Dinnon KH 3rd, Yount BL Jr et al. (2020). Trypsin Treatment Unlocks Barrier 360 for Zoonotic Bat Coronavirus Infection. J Virol 94: e01774-19.

361 Mukherjee S, Huda S, Sinha Babu SP (2019). Toll-like receptor polymorphism in host immune 362 response to infectious diseases: A review. Scand J Immunol 90:e12771.

363 Nelemans T, Kikkert M (2019). Viral Innate Immune Evasion and the Pathogenesis of Emerging 364 RNA Virus Infections. Viruses 11: 961.

365 Ou X, Liu Y, Lei X, Li P, Mi D, Ren L, Guo L, Guo R, Chen T, Hu J, Xiang Z, Mu Z, Chen X, 366 Chen J, Hu K, Jin Q, Wang J, Qian Z (2020). Characterization of spike glycoprotein of SARS367 CoV-2 on virus entry and its immune cross-reactivity with SARS-CoV. Nat Commun 11:1620. 368 Pickett BE, Greer DS, Zhang Y, Stewart L, Zhou L, Sun G, Gu Z, Kumar S, Zaremba S, Larsen 369 CN, Jen W, Klem EB, Scheuermann RH (2012). Virus pathogen database and analysis 370 resource (ViPR): a comprehensive bioinformatics database and analysis resource for the 371 coronavirus research community. Viruses 4: 3209-3226. 
372 Poulas K, Farsalinos K, Zanidis C (2020). Activation of TLR7 and Innate Immunity as an

373 Efficient Method Against COVID-19 Pandemic: Imiquimod as a Potential Therapy. Front

$374 \quad$ Immunol 11:1373.

375 Salata C, Calistri A, Parolin C, Palù G (2019). Coronaviruses: a paradigm of new emerging

$376 \quad$ zoonotic diseases. Pathog Dis 77: ftaa006.

377 Shah M, Anwar MA, Kim JH, Choi S (2016). Advances in Antiviral Therapies Targeting Toll-

378 like Receptors. Expert Opin Investig Drugs 25:437-453.

379 Streicher F, Jouvenet N (2019). Stimulation of Innate Immunity by Host and Viral RNAs. Trends

380 Immunol 40: 1134-1148.

381 To EE, Erlich J, Liong F, Luong R, Liong S, Bozinovski S, Seow HJ, O'Leary JJ, Brooks DA,

382 Vlahos R, Selemidis S (2019). Intranasal and epicutaneous administration of Toll-like receptor

3837 (TLR7) agonists provides protection against influenza A virus-induced morbidity in mice.

$384 \quad$ Sci Rep 9:2366.

385 van der Made CI, Simons A, Schuurs-Hoeijmakers J, van den Heuvel G, Mantere T, Kersten S,

386 van Deuren RC, Steehouwer M, van Reijmersdal SV, Jaeger M, Hofste T, Astuti G,

387 Corominas Galbany J, van der Schoot V, van der Hoeven H, Hagmolen Of Ten Have W, Klijn

388 E, van den Meer C, Fiddelaers J, de Mast Q, Bleeker-Rovers CP, Joosten LAB, Yntema HG,

389 Gilissen C, Nelen M, van der Meer JWM, Brunner HG, Netea MG, van de Veerdonk FL,

390 Hoischen A (2020). Presence of genetic variants among young men with severe COVID-19.

391 JAMA 324:1-11.

392 Via A, Uyar B, Brun C, Zanzoni A (2015). How pathogens use linear motifs to perturb host cell

393 networks. Trends Biochem Sci 40: 36-48. 
394 Vierbuchen T, Stein K, Heine H (2019). RNA is taking its Toll: Impact of RNA-specific Toll395 like receptors on health and disease. Allergy 74: 223-235.

396 Wong LY, Lui PY, Jin DY (2016). A molecular arms race between host innate antiviral response 397 and emerging human coronaviruses. Virol Sin 31: 12-23.

398 Xia S, Liu M, Wang C, Xu W, Lan Q, Feng S, Qi F, Bao L, Du L, Liu S, Qin C, Sun F, Shi Z, 399 Zhu Y, Jiang S, Lu L (2020). Inhibition of SARS-CoV-2 (previously 2019-nCoV) infection by 400 a highly potent pan-coronavirus fusion inhibitor targeting its spike protein that harbors a high 401 capacity to mediate membrane fusion. Cell Res 30: 343-355.

402 Yang CW (2012a). A comparative study of short linear motif compositions of the influenza A $403 \quad$ virus ribonucleoproteins. PLoS One 7: e38637.

404 Yang CW, Chen SM (2012b). A comparative study of human TLR 7/8 stimulatory trimer 405 compositions in influenza A viral genomes. PLoS One 7: e30751.

406 Yang CW, Chen MF (2020). Composition of human-specific slow codons and slow di-codons in 407 SARS-CoV and 2019-nCoV are lower than other coronaviruses suggesting a faster protein 408 synthesis rate of SARS-CoV and 2019-nCoV. J Microbiol Immunol Infect 53: 419-424. 409 Ye ZW, Yuan S, Yuen KS, Fung SY, Chan CP, Jin DY (2020). Zoonotic origins of human 410 coronaviruses. Int J Biol Sci 16: 1686-1697.

411 Yokota S, Okabayashi T, Fujii N (2010). The battle between virus and host: modulation of Toll412 like receptor signaling pathways by virus infection. Mediators Inflamm 2010: 184328. 413 Zhang Z, Ohto U, Shibata T, Taoka M, Yamauchi Y, Sato R, Shukla NM, David SA, Isobe T, 414 Miyake K, Shimizu T (2018). Structural analyses of Toll-like receptor 7 reveal detailed RNA 415 sequence specificity and recognition mechanism of agonistic ligands. Cell Rep 25:3371$416 \quad 3381 . e 5$. 
417 Zheng J (2020). SARS-CoV-2: an emerging coronavirus that causes a clobal threat. Int J Biol Sci 418 16: 1678-1685.

419 Zhou M, Zhang X, Qu J (2020). Coronavirus disease 2019 (COVID-19): a clinical update. Front 420 Med 14: 126-135.

421

422 Table Caption

423

424 Table 1. Coefficients of logistic regression from the 10-fold cross-validation and from all data.

425 Table 2. Overall agreements of the logistic regression model compared with the other six models. 426

427 Figure Captions

428

429 Fig. 1. Design rationale for evaluating the interactions between viral RNAs and human TLR 7/8

430 by analyzing the human TLR 7/8 stimulatory triribonucleotide composition of viral RNAs.

431

432 Fig. 2. Comparison of analysis results from the conventional phylogenetic method and the 433 weight triribonucleotide compositions proposed in this study (A) Phylogenetic analysis of full434 length genomic sequences of representative coronaviruses infecting human hosts. The number of 435 each branch obtained from 1000 bootstrapping is shown. The accession numbers indicate the 436 representative genomic sequences used in this analysis. (B) Comparison of triribonucleotide 437 compositions of coronaviruses infecting human hosts. 229E: human coronavirus 229E. NL63:

438 human coronavirus NL63. HKU1: human coronavirus HKU1. OC43: human coronavirus OC43. 439 SARS: severe acute respiratory syndrome coronavirus (SARS-CoV), MERS: Middle East 
440 respiratory syndrome coronavirus (MERS-CoV). SARS2: severe acute respiratory syndrome 441 coronavirus 2 (SARS-CoV-2). Other: unclassified coronaviruses. The lowercase r notations 442 indicate the complementary (-) strands of genomic RNAs (replication intermediates). The red 443 stars “*” indicate human TLR 7/8 stimulatory triribonucleotides.

444

445 Fig. 3. Human TLR 7/8-stimulating scores of coronavirus genomic (positive strand) RNAs. 446 Score S: human TLR 7/8 stimulating score. Score N: human TLR 7/8 non-stimulating score. 447 229E: human coronavirus 229E. NL63: human coronavirus NL63. HKU1: human coronavirus 448 HKU1. OC43: human coronavirus OC43. SARS: severe acute respiratory syndrome coronavirus 449 (SARS-CoV), MERS: Middle East respiratory syndrome (MERS-CoV). SARS2: severe acute 450 respiratory syndrome coronavirus 2 (SARS-CoV-2).

451

452 Fig. 4. Human TLR 7/8-stimulating scores of the complementary (negative) strands of 453 coronavirus genomic RNAs (replication intermediates). Score S: human TLR 7/8-stimulating 454 score. Score N: human TLR 7/8 non-stimulating scores 229E: human coronavirus 229E. NL63:

455 human coronavirus NL63. HKU1: human coronavirus HKU1. OC43: human coronavirus OC43. 456 SARS: severe acute respiratory syndrome coronavirus (SARS-CoV), MERS: Middle East 457 respiratory syndrome (MERS-CoV). SARS2: severe acute respiratory syndrome coronavirus 2 458 (SARS2-CoV-2).

459

460 Fig. 5. Prediction of human TLR 7/8-stimulating ability of coronaviruses from mammalian and 461 avian hosts by the logistic regression model constructed from human coronavirus data. $\mathrm{P} \cong 1$ 462 indicates a human TLR 7/8-stimulating ability equivalent to that of 229E, NL63, OC43 and 


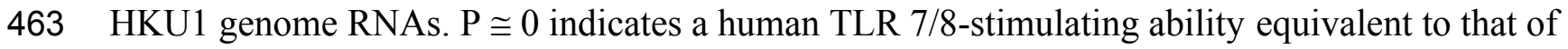
464 MERS-CoV, SARS-CoV and SARS-CoV-2 genomic RNAs. The number of viruses analyzed is 465 shown in the denominators. Avian: birds other than chickens.

466

467 


\section{Figure 1}

Design rationale for evaluating the interactions between viral RNAs and human toll-like receptors $7 / 8$

Fig. 1. Design rationale for evaluating the interactions between viral RNAs and human TLR $7 / 8$ by analyzing the human TLR 7/8 stimulatory triribonucleotide composition of viral RNAs. 
A tri-mer weight vector derived from human TLR 7/8 stimulatory oligoribonucleotides
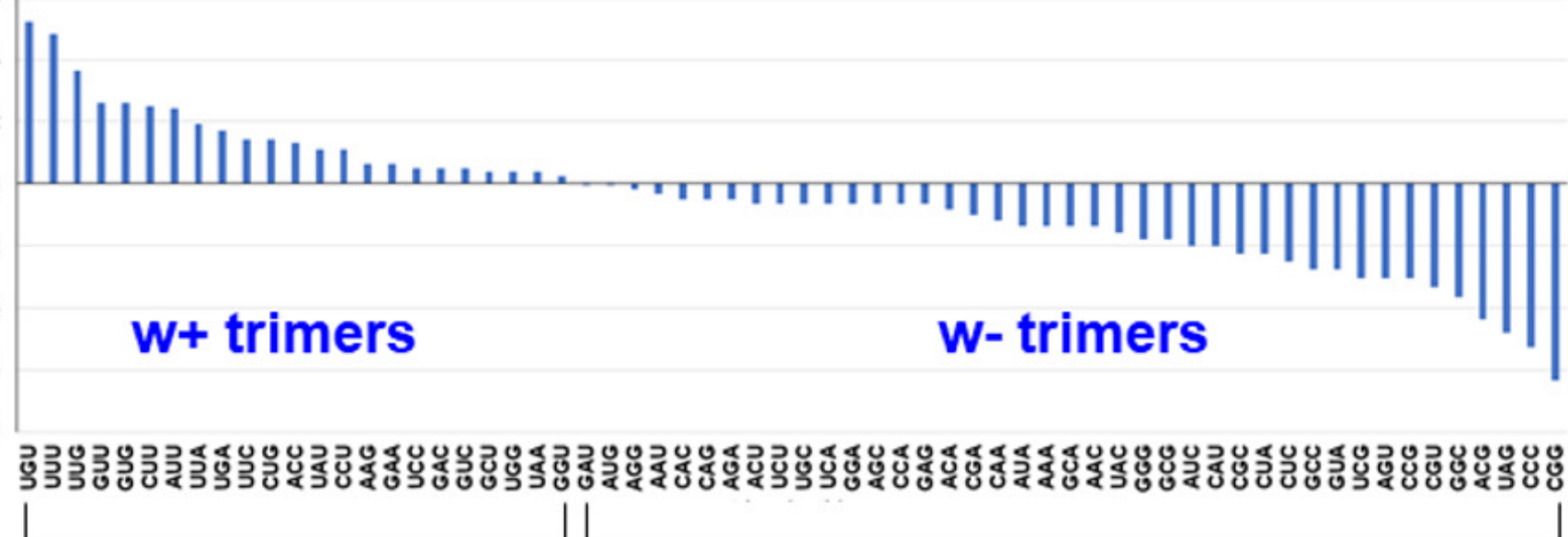

Human TLR $7 / 8$

stimulatory trimer

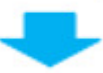

$\Sigma$ (composition of trimers in viral RNA $\times \mathbf{w}^{+}$)

Weighted Scores

Virus 1: high Score S, high Score $\mathbf{N}$ Viral RNA with many $w+$ trimers

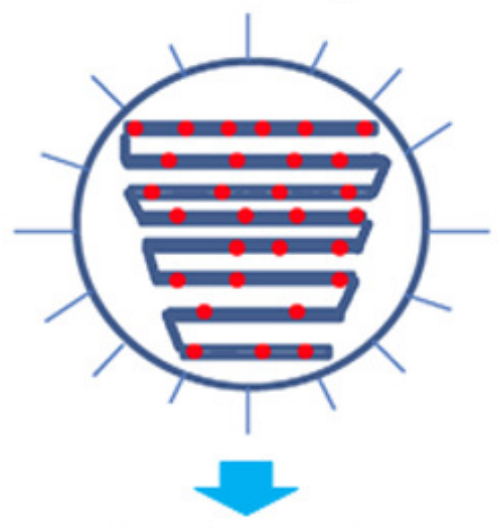

Strong stimulatory interactions with human TLR 7/8

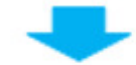

Human TLR 7/8 nonstimulatory trimer $\Sigma$ (composition of trimers in viral RNA $\times$ w-)
Virus 2: low Score S, low Score N Viral RNA with few $w+$ trimers

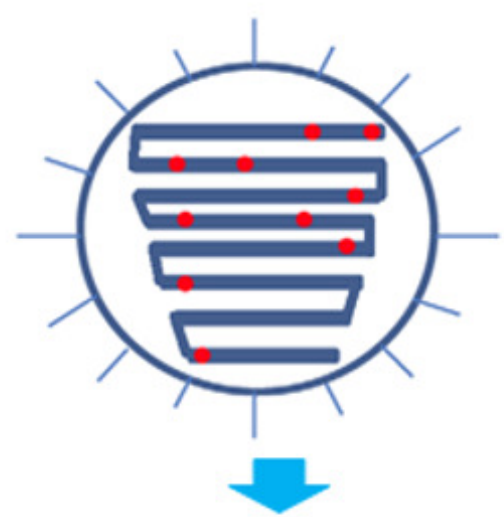

Weak stimulatory interactions with human TLR 7/8

Different ability to evade from human TLR $7 / 8$ dependent innate immunity 


\section{Figure 2}

Comparison of sequence and ribonucleotide trimer compositions of coronaviruses infect human hosts

Fig. 2. Comparison of analysis results from the conventional phylogenetic method and the weight triribonucleotide compositions proposed in this study (A) Phylogenetic analysis of fulllength genomic sequences of representative coronaviruses infecting human hosts. The number of each branch obtained from 1000 bootstrapping is shown. The accession numbers indicate the representative genomic sequences used in this analysis. (B) Comparison of triribonucleotide compositions of coronaviruses infecting human hosts. 229E: human coronavirus 229E. NL63: human coronavirus NL63. HKU1: human coronavirus HKU1. OC43: human coronavirus OC43. SARS: severe acute respiratory syndrome coronavirus (SARS-CoV), MERS: Middle East respiratory syndrome coronavirus (MERS-CoV). SARS2: severe acute respiratory syndrome coronavirus 2 (SARS-CoV-2). Other: unclassified coronaviruses. The lowercase $r$ notations indicate the complementary (-) strands of genomic RNAs (replication intermediates). The red stars "*” indicate human TLR 7/8 stimulatory triribonucleotides. 

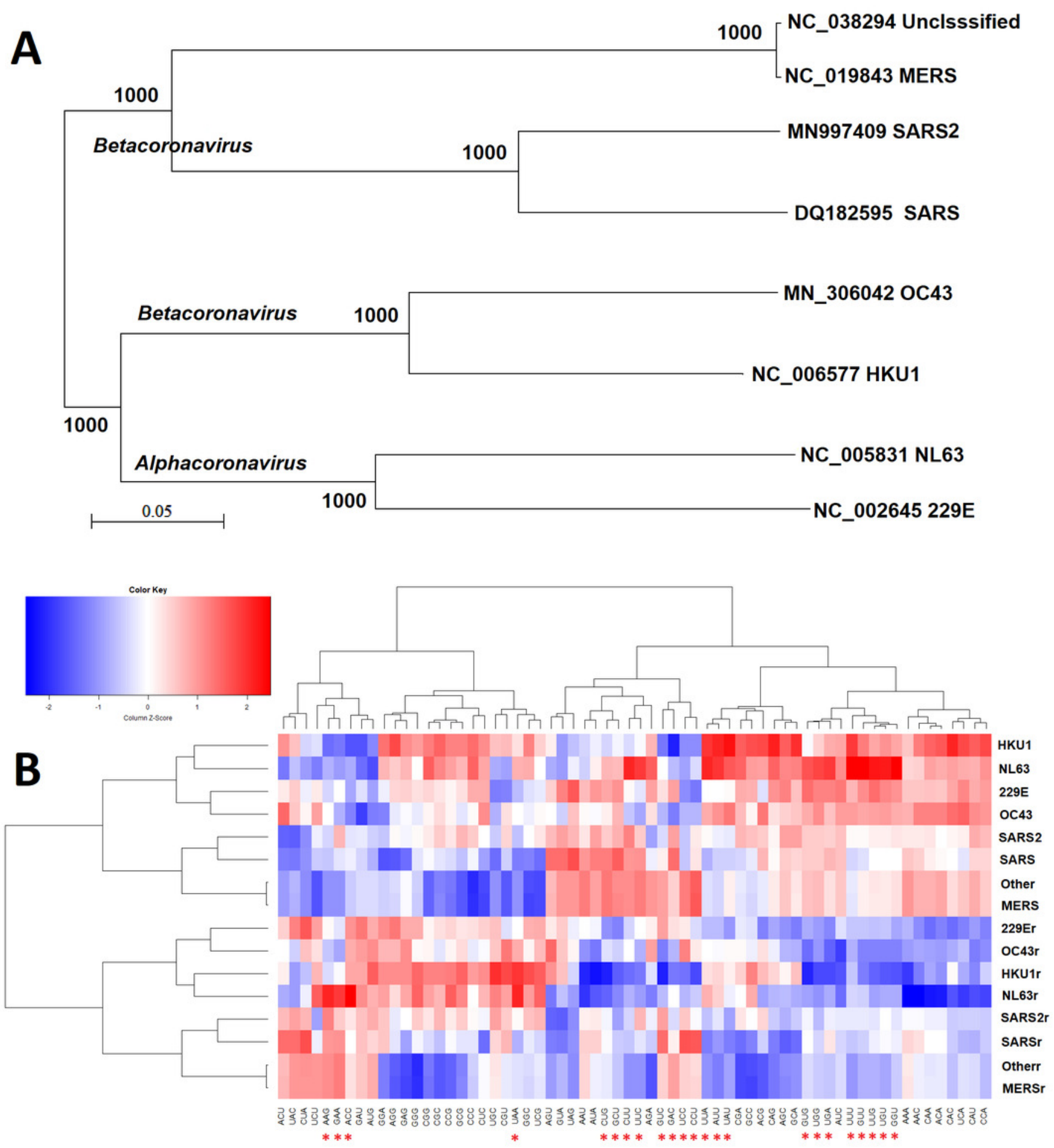


\section{Figure 3}

The human toll-like receptor 7/8-stimulating scores of coronavirus genome (positive strand) RNAs.

Fig. 3. Human TLR 7/8-stimulating scores of coronavirus genomic (positive strand) RNAs. Score S: human TLR 7/8 stimulating score. Score N: human TLR 7/8 non-stimulating score. 229E: human coronavirus 229E. NL63: human coronavirus NL63. HKU1: human coronavirus HKU1. OC43: human coronavirus OC43. SARS: severe acute respiratory syndrome coronavirus (SARS-CoV), MERS: Middle East respiratory syndrome (MERS-CoV). SARS2: severe acute respiratory syndrome coronavirus 2 (SARS-CoV-2). 


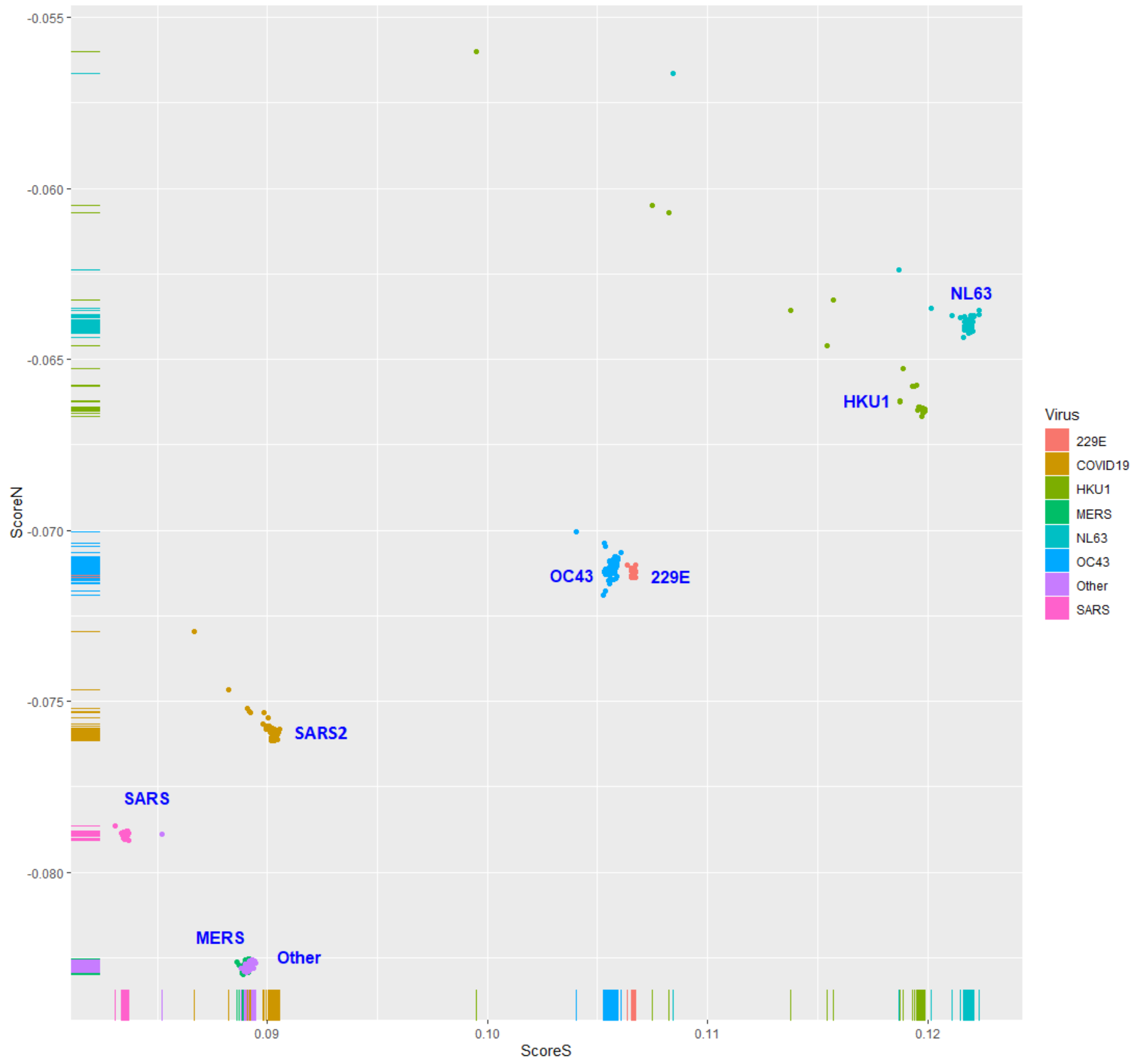




\section{Figure 4}

The human toll-like receptor 7/8-stimulating scores of the complementary (negative) strand of coronavirus genomic RNAs (replication intermediates).

Fig. 4. Human TLR 7/8-stimulating scores of the complementary (negative) strands of coronavirus genomic RNAs (replication intermediates). Score S: human TLR 7/8-stimulating score. Score N: human TLR 7/8 non-stimulating scores 229E: human coronavirus 229E. NL63: human coronavirus NL63. HKU1: human coronavirus HKU1. OC43: human coronavirus OC43. SARS: severe acute respiratory syndrome coronavirus (SARS-CoV), MERS: Middle East respiratory syndrome (MERS-CoV). SARS2: severe acute respiratory syndrome coronavirus 2 (SARS2-CoV-2). 


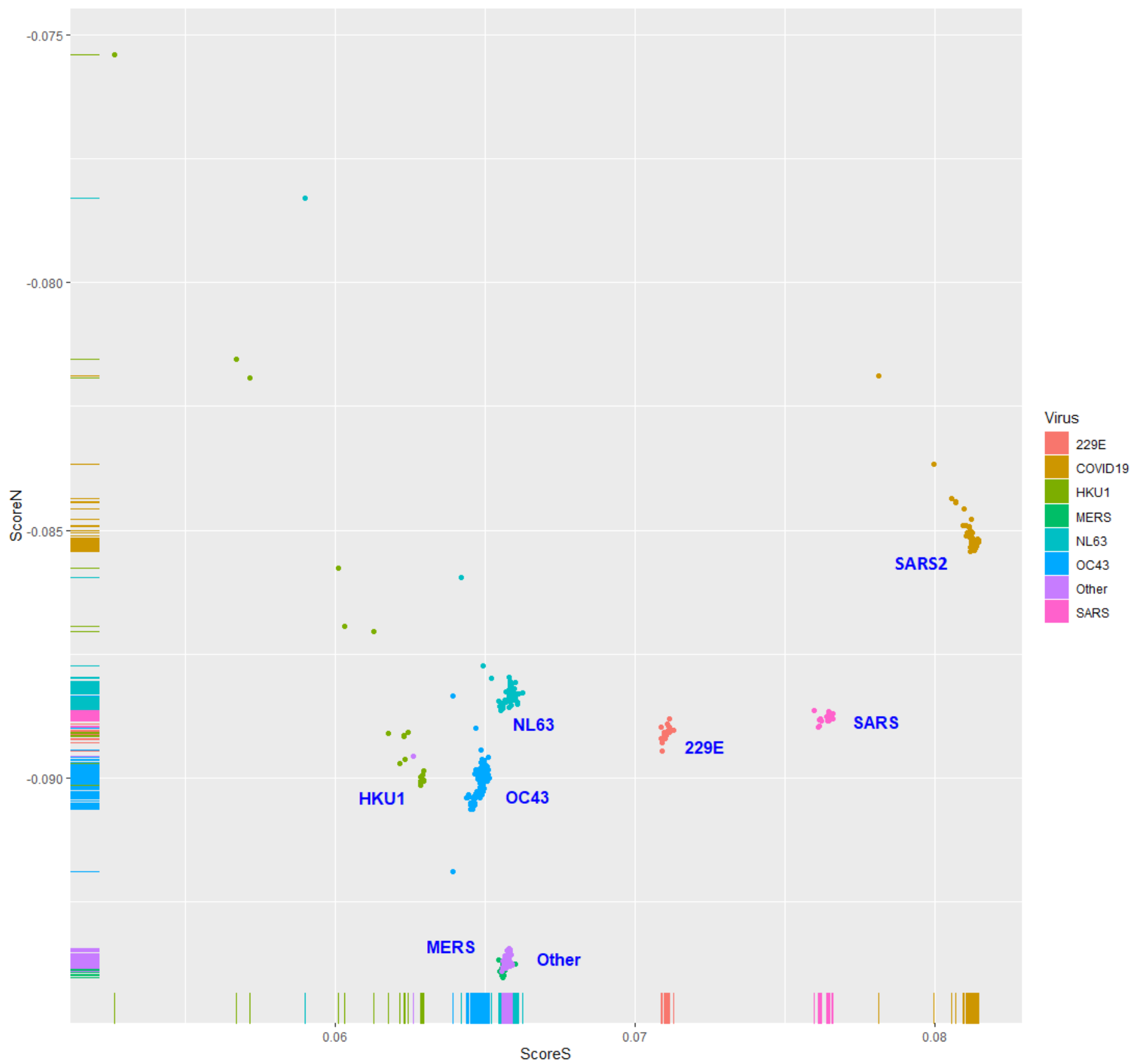




\section{Figure 5}

Prediction of human toll-like receptor 7/8-stimulating ability of coronaviruses from mammalian and avian hosts by the logistic regression model constructed from human coronavirus data.

Fig. 5. Prediction of human TLR 7/8-stimulating ability of coronaviruses from mammalian and avian hosts by the logistic regression model constructed from human coronavirus data. $\mathrm{P} \cong 1$ indicates a human TLR 7/8-stimulating ability equivalent to that of 229E, NL63, OC43 and HKU1 genome RNAs. $P \cong 0$ indicates a human TLR 7/8-stimulating ability equivalent to that of MERS-CoV, SARS-COV and SARS-CoV-2 genomic RNAs. The number of viruses analyzed is shown in the denominators. Avian: birds other than chickens. 


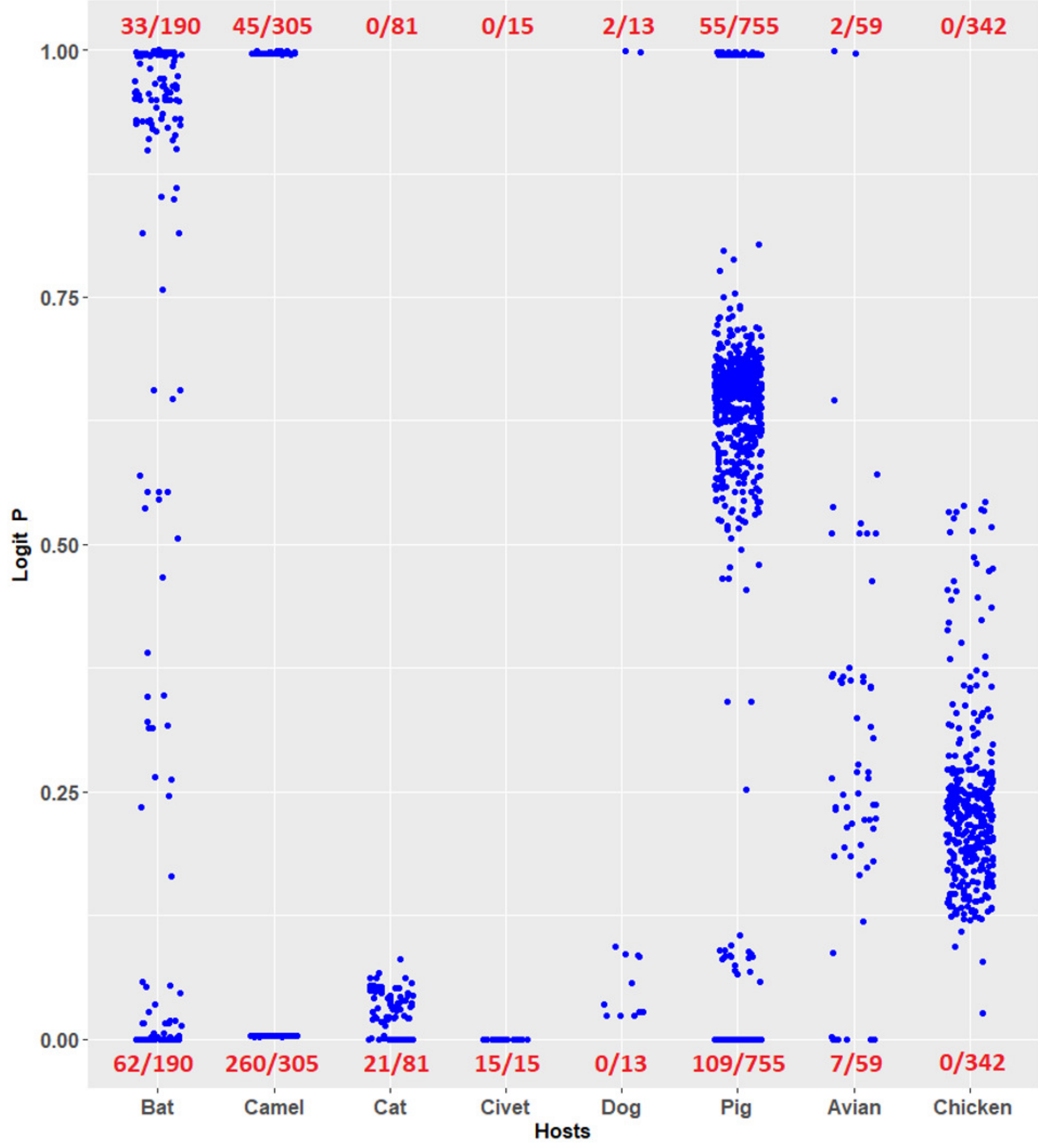




\section{Table $\mathbf{1}$ (on next page)}

Coefficients of logistic regression from the 10 -fold cross-validation and from all data.

Table 1. Coefficients of logistic regression from a 10-fold cross validation and all data. 
1 Table 1. Coefficients of logistic regression from the 10-fold cross-validation and from all data. 2

\begin{tabular}{|c|c|c|c|c|c|c|c|}
\hline & $\begin{array}{l}\text { Intercept } \\
\left(\beta_{0}\right)\end{array}$ & p-value & $\mathrm{S}\left(\beta_{l}\right)$ & p-value & sensitivity & specificity & accuracy \\
\hline Set01 & -69.14 & $\begin{array}{l}7.48 \mathrm{e}- \\
13\end{array}$ & 711.33 & $\begin{array}{l}1.10 \mathrm{e}- \\
11\end{array}$ & 1 & 1 & 1 \\
\hline Set02 & -78.48 & $\begin{array}{l}1.92 \mathrm{e}- \\
07\end{array}$ & 809.73 & $\begin{array}{l}8.88 \mathrm{e}- \\
07\end{array}$ & 1 & 0.96 & 0.99 \\
\hline Set03 & -69.12 & $\begin{array}{l}7.92 \mathrm{e}- \\
13\end{array}$ & 711.08 & $\begin{array}{l}1.17 \mathrm{e}- \\
11\end{array}$ & 1 & 1 & 1 \\
\hline Set04 & -69.05 & $\begin{array}{l}8.11 \mathrm{e}- \\
13\end{array}$ & 710.35 & $\begin{array}{l}1.20 \mathrm{e}- \\
11\end{array}$ & 1 & 1 & 1 \\
\hline Set05 & -69.05 & $\begin{array}{l}8.11 \mathrm{e}- \\
13\end{array}$ & 710.35 & $\begin{array}{l}1.20 \mathrm{e}- \\
11\end{array}$ & 1 & 1 & 1 \\
\hline Set06 & -69.15 & $\begin{array}{l}7.56 \mathrm{e}- \\
13\end{array}$ & 711.45 & $\begin{array}{l}1.12 \mathrm{e}- \\
11\end{array}$ & 1 & 1 & 1 \\
\hline Set07 & -69.16 & $\begin{array}{l}7.47 \mathrm{e}- \\
13\end{array}$ & 711.58 & $\begin{array}{l}1.10 \mathrm{e}- \\
11\end{array}$ & 1 & 0.96 & 0.99 \\
\hline Set08 & -67.95 & $\begin{array}{l}1.07 e- \\
13\end{array}$ & 697.92 & $\begin{array}{l}1.96 \mathrm{e}- \\
12\end{array}$ & 1 & 1 & 1 \\
\hline Set09 & -69.16 & $\begin{array}{l}7.50 e- \\
13 \\
\end{array}$ & 711.56 & $\begin{array}{l}1.11 \mathrm{e}- \\
11\end{array}$ & 1 & 1 & 1 \\
\hline Set10 & -79.87 & $\begin{array}{l}4.31 \mathrm{e}- \\
09\end{array}$ & 819.50 & $\begin{array}{l}2.22 \mathrm{e}- \\
08\end{array}$ & 1 & 0.96 & 0.99 \\
\hline Mean & -71.01 & & 730.49 & & & & \\
\hline Std & 4.32 & & 44.59 & & & & \\
\hline All & -69.05 & $\begin{array}{l}8.11 \mathrm{e}- \\
13\end{array}$ & 710.36 & $\begin{array}{l}1.20 \mathrm{e}- \\
11 \\
\end{array}$ & & & \\
\hline
\end{tabular}

3 Set01-Set10 using 9/10 data (as training data) was used to do regression, and 1/10 data as test 4 data. All: 10/10 data was used to do regression. 


\section{Table 2 (on next page)}

Overall agreements of the logistic regression model compared with the other six models.

Table 2. Overall agreements of the logistic regression model compared with the other six models. 
1 Table 2. Overall agreements of the logistic regression model compared with the other six models. 2

\begin{tabular}{|l|l|l|l|l|l|l|}
\hline & lda & qda & nb & svmL & svmP & svmR \\
\hline Avian & 0.86 & 0.34 & 0.86 & 0.88 & 0.88 & 0.17 \\
\hline Bat & 0.86 & 0.93 & 0.88 & 0.96 & 0.96 & 0.65 \\
\hline Camel & 1 & 1 & 1 & 1 & 1 & 0.96 \\
\hline Cat & 1 & 0.81 & 0.95 & 1 & 1 & 0.01 \\
\hline Chicken & 0.97 & 0.35 & 0.97 & 0.97 & 0.97 & 0.03 \\
\hline Civet & 1 & 1 & 1 & 1 & 1 & 0.94 \\
\hline Dog & 1 & 1 & 1 & 1 & 1 & 0.15 \\
\hline pig & 0.25 & 0.84 & 0.26 & 0.81 & 0.78 & 0.82 \\
\hline
\end{tabular}

3 lda: Linear discriminant analysis, qda: Quadratic discriminant analysis, nb: Naive Bayes, svmL: 4 SVM linear classifier, svmP: SVM polynomial classifier, svmR: SVM radial classifier 